\title{
Comparison of Methods to Quantify Volume During Resistance Exercise
}

\author{
Jeffrey M. McBride, Grant O. McCaulley, Prue Cormie, \\ James L. Nuzzo, Michael J. Cavill, and N Travis Triplett \\ Neuromuscular Laboratory, Department of Health, Leisure \& Exercise Science, \\ Appalachian State University, Boone, North Carolina
}

\begin{abstract}
McBride, JM, McCaulley, GO, Cormie, P, Nuzzo, JL, Cavill, MJ, and Triplett, NT. Comparison of methods to quantify volume during resistance exercise. J Strength Cond Res 23(1): 106$110,2009-T h e$ purpose of this investigation was to compare 4 different methods of calculating volume when comparing resistance exercise protocols of varying intensities. Ten Appalachian State University students experienced in resistance exercise completed 3 different resistance exercise protocols on different days using a randomized, crossover design, with 1 week of rest between each protocol. The protocols included 1) hypertrophy: 4 sets of 10 repetitions in the squat at $75 \%$ of a 1 -repetition maximum (1RM) (90-second rest periods); 2) strength: 11 sets of 3 repetitions at $90 \% 1 \mathrm{RM}$ (5-minute rest periods); and 3) power: 8 sets of 6 repetitions of jump squats at $0 \% 1 \mathrm{RM}$ (3-minute rest periods). The volume of resistance exercise completed during each protocol was determined with 4 different methods: 1) volume load (VL) (repetitions [no.] $\times$ external load [kg]); 2) maximum dynamic strength volume load (MDSVL) (repetitions [no.] $\times$ [body mass - shank mass (kg) + external load (kg)]); 3) time under tension (TUT) (eccentric time +milliseconds] + concentric time +milliseconds]); and 4) total work (TW) (force $[N] \times$ displacement $[\mathrm{m}])$. The volumes differed significantly $(p<0.05)$ between hypertrophy and strength in comparison with the power protocol when VL and MDSVL were used to determine the volume of resistance exercise completed. Furthermore, significant differences in TUT existed between all 3 resistance exercise protocols. The TW calculated was not significantly different between the 3 protocols. These data imply that each
\end{abstract}

Address correspondence to Jeffrey M. McBride, mcbridejm@appstate. edu.

23(1)/106-110

Journal of Strength and Conditioning Research

(C) 2009 National Strength and Conditioning Association method examined results in substantially different values when comparing various resistance exercise protocols involving different levels of intensity.

Key WorDs time under tension, squat, jump squat, volume load

INTRODUCTION

1 ariation in the volume of resistance exercise completed can be a potent stimulus for positive adaptations and is critical for the researcher and the practitioner to quantify and monitor $(7,11,15)$. However, a standardized method to determine volume during resistance exercise has not been defined in the current literature (7). This is attributable to the complex nature of variables that encapsulate resistance exercise protocols (i.e., intensity, repetitions, sets). Several methods have been used to determine volume in the current literature including volume load (VL), maximum dynamic strength volume load (MDSVL), time under tension (TUT), and total work (TW).

Volume load (repetitions [no.] $\times$ external load [kg]) has been used to monitor athletes (9) and to equate resistance exercise volumes between experimental conditions $(1,2,11)$. This method is convenient because load and repetitions can be easily monitored. However, VL is inadequate for calculating volume when performing movements with no external load (i.e., body mass-only exercises). The MDSVL method (repetitions [no.] $\times$ [body - shank mass $(\mathrm{kg})+$ external load $(\mathrm{kg})]$ ) gives researchers and practitioners a way to quantify volume during resistance exercise when no external load is present; this method has been used to calculate volume during power-type resistance exercise such as body mass jump squats (5). However, neither VL nor MDSVL accounts for variables such as actual force exerted and barbell displacement, which may more accurately reflect the exercise stimulus.

Volume determination using TUT is another method often reported in the literature $(7,8,10,16)$. This method involves monitoring repetition time to perform eccentric and/or concentric actions during the exercise (16). Tran et al. (16) varied load intensities and concentric phase contraction speed (seconds) to elicit differences in TUT. The

106 Journal of Strength and Conditioning Research 
Journal of Strength and Conditioning Research" $\mid$ www.nsca-jscr.org

shortcomings of this method are that it does not account for body mass, external load, actual force produced, or displacement of the barbell. The TW method (force $[\mathrm{N}] \times$ displacement $[\mathrm{m}]$ ) has also been used to quantify resistance exercise volume $(4,13,14)$. This method accounts for actual force exerted and displacement of the center of mass or barbell during the exercise.

The arrangement of resistance exercise protocols has been observed to dictate the chronic adaptation desired $(2,11)$. By using targeted intensities and rest periods, specific training goals (e.g., hypertrophy, strength and power) may be optimized (6). However, the effectiveness of a specific resistance exercise protocol is also related to the volume of exercise completed. To isolate the influence of intensity of a resistance exercise protocol and its effect on strength, volume must be accounted for. Questions remain regarding the legitimacy of various methods of volume quantification when comparing different types of resistance exercise protocols. Therefore, the purpose of this investigation was to calculate volume using 4 methods (VL, MDSVL, TUT, TW) for 3 different types of resistance exercise protocols with varying intensities.

\section{Methods}

\section{Experimental Approach to the Problem}

This investigation used a randomized crossover design to examine the effectiveness of different methods of calculating volume when comparing resistance exercise sessions of varied intensities. Differences between the experimental protocols for each method of volume calculation were determined. The protocols included 1) hypertrophy, 2) strength, and 3) power. The volume of resistance exercise completed during each protocol was determined with 4 different methods: 1) VL, 2) MDSVL, 3) TW, and 4) TUT.

\section{Subjects}

For this study, 10 subjects (all men; age: $21.8 \pm 1.9$ years; height: $176.3 \pm 7.0 \mathrm{~cm}$; body mass: $92.4 \pm 9.5 \mathrm{~kg}$; body fat: $13.2 \pm 4.2 \%$; 1-repetition maximum [1RM] squat: $170.8 \pm$ $24.9 \mathrm{~kg} ; 1 \mathrm{RM}$ squat:body mass ratio $1.9 \pm 0.2)$ were recruited. The subjects were chosen for their experience with resistance exercise and proficiency in the back squat exercise. The subjects were instructed to refrain from any intense lowerbody training for 24 hours before testing and throughout the training period. The data from this investigation were part of a larger cross-sectional investigation that assessed the acute effects of resistance exercise protocols of varied intensity on neuromuscular and endocrine responses. The participants were notified about the potential risks involved and gave their written informed consent. This study was approved by the institutional review board at Appalachian State University.

\section{Strength Testing}

Baseline strength levels were determined by assessing a 1RM for the back squat exercise as previously described $(12,13)$. Only back squats that achieved a knee angle of $90^{\circ}$ were considered successful attempts. Warm-up sets using loads of 50,70 , and $90 \%$ of an estimated 1RM were completed, followed by $2-4$ maximal attempts until volitional failure was achieved.

\section{Experimental Protocols}

The subjects completed 3 experimental protocols. The experimental protocols were completed in a randomized fashion on separate days. One week was allowed between each treatment. The hypertrophy protocol included 4 sets of 10 repetitions of the back squat at 75\% $1 \mathrm{RM}$ with a 90 -second rest period between each set. The strength protocol included 11 sets of 3 repetitions of the back squat at 90\% 1RM with a 5 -minute rest period between sets. The power protocol included 8 sets of 6 jump squats at body mass (i.e., $0-\mathrm{kg}$ external load) with a 3 -minute rest period between sets.

\section{Volume Calculations}

The VL was determined by the multiplication of total repetitions completed and external load (repetitions [no.] $\times$ external load $[\mathrm{kg}]$ ). The MDSVL method used the following equation: (repetitions [no.] $\times$ [body mass - shank mass $(\mathrm{kg})$ + external load (kg)]). The TUT was assessed by determining the amount of time (milliseconds) spent in the eccentric and concentric phases for every successful back squat and jump squat repetition. In addition, TUT was calculated per repetition in seconds. The TW in joules was calculated by adding the eccentric and concentric work for each repetition. Eccentric and concentric work were determined by integrating the area under the curve of the force-displacement graphs for the eccentric and concentric phases of each repetition. Data for calculating TUTand TW involved vertical and horizontal bar displacements, which were measured by 2 linear position transducers $(3-5,14)$. Vertical ground reaction force was measured using a force plate (AMTI, BP6001200, Watertown, Mass). Analog data (2 linear position transducers and 1 force plate) were recorded using a shielded BNC adapter chassis (National Instruments, BNC-2090, Austin, Tex) and an A/D card (National Instruments, NI PCI-6014) at $1000 \mathrm{~Hz}$. Custom-written programs using LabVIEW (National Instruments, version 7.1) were used for recording and analyzing the data $(3-5,14)$.

\section{Statistical Analyses}

Standard statistical methods were used to determine mean and $S D$. Differences between the experimental protocols for each method of volume calculation were determined using a general linear regression model (SPSS version 13.0). The significance level was set at $p \leq 0.05$ for this investigation.

\section{Results}

There was no significant difference between VL when comparing the hypertrophy and strength protocols $(p \leq$ 0.05) (Figure 1). The VL for the power protocol was zero because no external load was used; thus, no statistical comparison was possible. There was no significant difference 


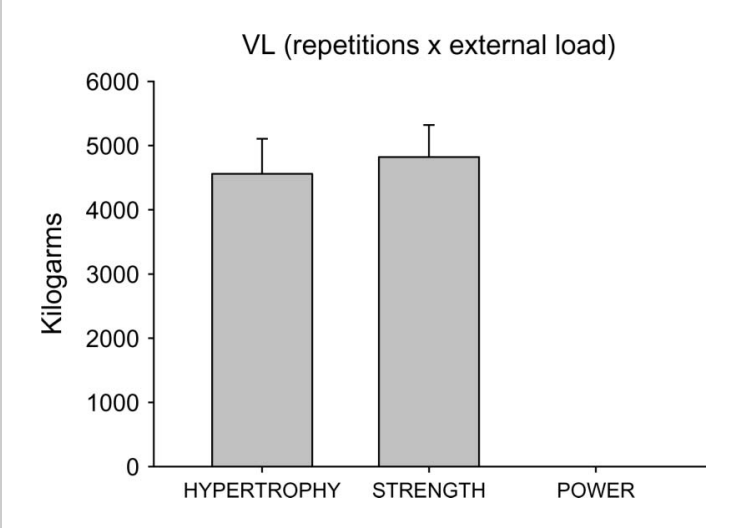

Figure 1. A comparison of the volume load $(\mathrm{VL})$ in kilograms calculated between hypertrophy, strength, and power-type resistance exercise. No value can be assigned to power because of the absence of an external load.

between the hypertrophy and strength protocols when comparing MDSVL (Figure 2). However, the MDSVL in the power protocol was significantly lower than in both the hypertrophy and strength protocols. The TUT was significantly different between all 3 protocols (strength > hypertrophy $>$ power) in terms of total TUT (Figure 3 ) and TUT per repetition (Figure 4). The difference in repetition speed was also evident in the significant difference in time per repetition between the strength $>$ hypertrophy $>$ power protocols (strength $=2.9$ seconds, hypertrophy $=2.3$ seconds, power $=0.74$ seconds). Values for TW were not significantly different between the 3 protocols (Figure 5).

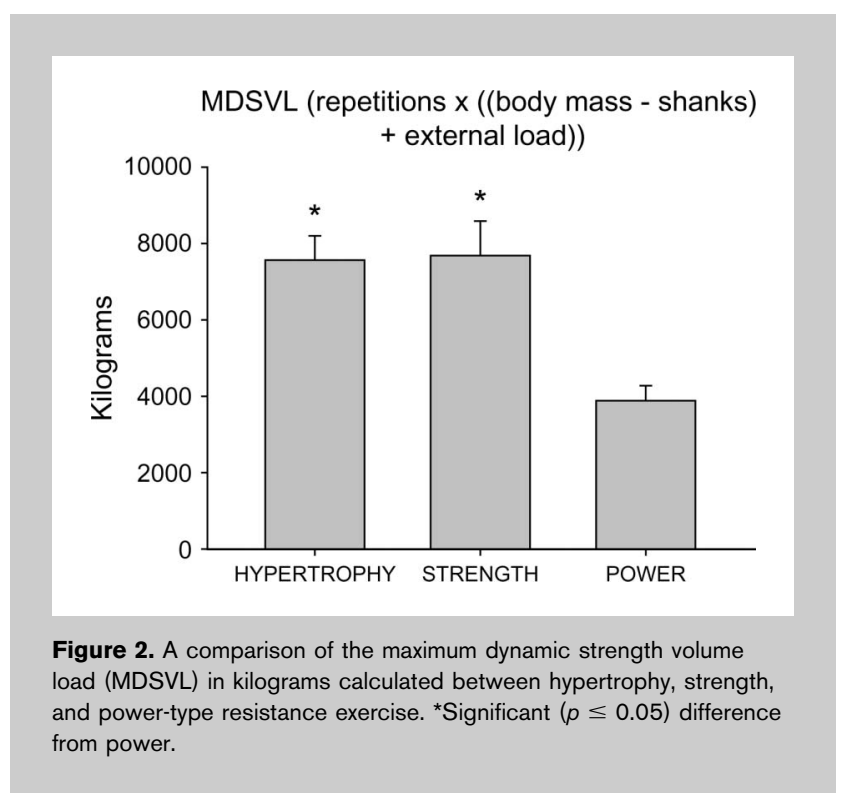

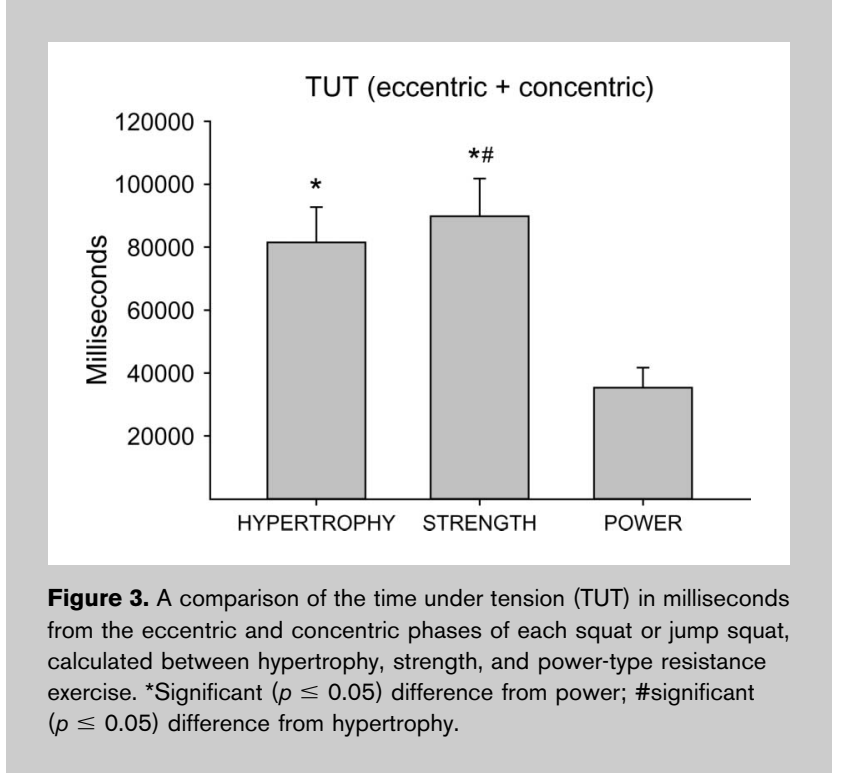

\section{Discussion}

The current data illustrate that analysis of TW provides the most valid determination of resistance exercise volume, which was not significantly different between the 3 resistance exercise protocols. The other methods used (VL, MDSVL, TUT) resulted in discrepancies in the amount of volume completed in each protocol. The VL method is flawed because it cannot express volume during exercises with no external mass. The MDSVL method underestimates the volume completed during the power protocol because of the underestimation of the actual amount of force exerted during explosive exercises. The TUT method may be a valuable

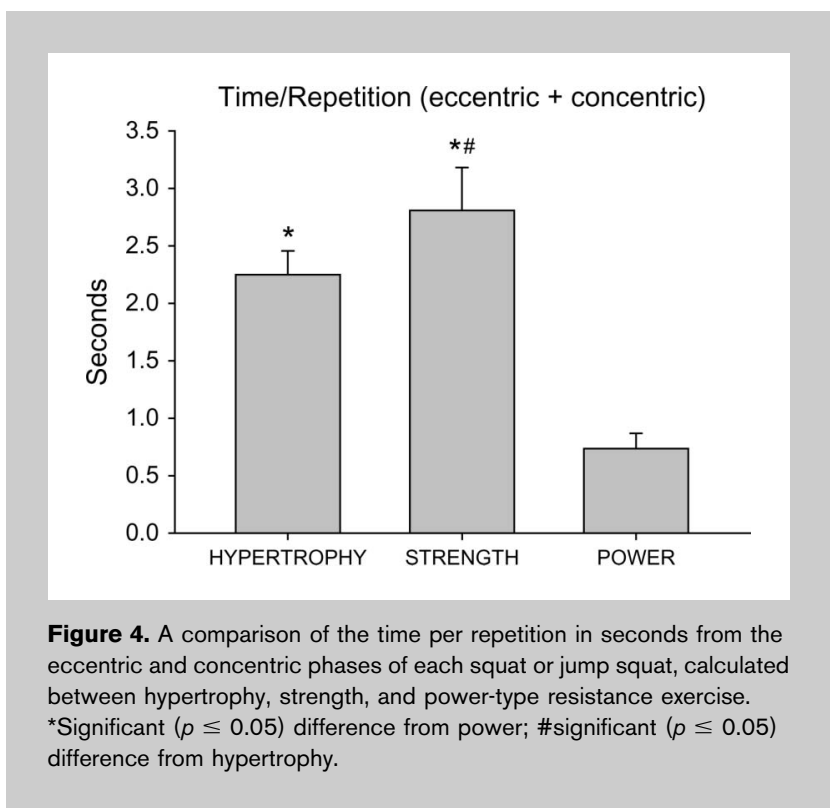




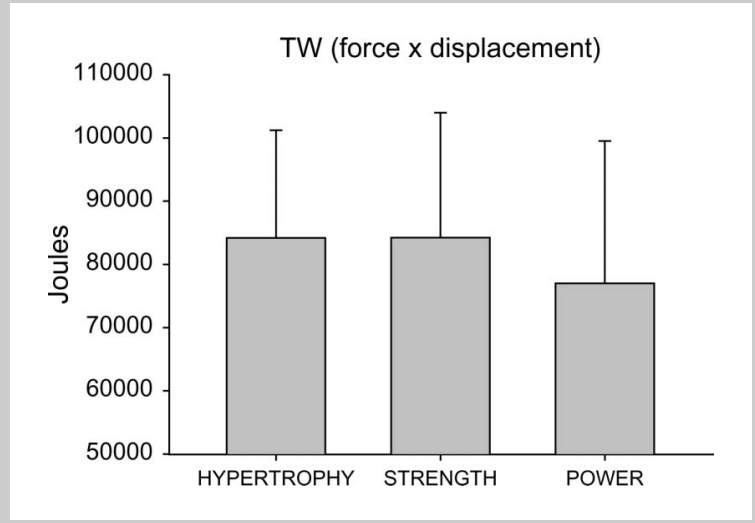

Figure 5. A comparison of the total work (TW) in joules calculated between hypertrophy, strength, and power-type resistance exercise.

variable to consider in terms of time spent developing force, but, in itself, it does not adequately express the stimulus in terms of actual force exerted and displacement of the barbell.

Determination of volume from the VL equation can be advantageous because of its simplicity in calculation of volume from a hypertrophy or strength resistance training protocol. However, one disadvantage of VL is the inability to equate volume when a $0-\mathrm{kg}$ load is used. This may be problematic for power-based training programs because current research has shown that jump squat power output is optimized when using a $0-\mathrm{kg}$ external load (5). Another limitation of VL is that the TUT cannot be accurately assessed in a given set of repetitions. The velocity at which concentric and eccentric phases are performed in a repetition has been shown to significantly affect the amount of total work performed (16).

Determination of volume from MDSVL has been an uncertainty in the current literature. Recently, researchers have disputed the inclusion of the entire body mass in the system mass used for volume calculations or whether body mass minus the shank mass should be used (5). Upper-body movements are primarily executed independently of the lower body and, therefore, do not require the inclusion of body mass. However, the demands of a lower-body movement such as the squat or jump squat require the athlete to accelerate and decelerate the external load and body mass, leading the authors to believe that body mass should be included regardless of the methodology. The results from the current investigation indicate the ability of MDSVL to quantify volume in lower-body resistance exercise at all loads including $0 \mathrm{~kg}$. Whereas MDSVL seems to be an effective method to determine volume because it accounts for body mass, it still fails to account for TUT, velocity of the movement, and displacement.

Previous research quantifying TUT has identified the cumulative time muscles are under tension as a key variable in eliciting hypertrophy in response to resistance exercise (8). Although volume determination from TUT is easy to measure, it does not directly account for load and is difficult to quantify during high-velocity movements. Furthermore, the data from the current investigation indicate significant differences between TUT even when the amount of total work performed between the 3 protocols is not significantly different. This was most evident in the power protocol in which the jump squat was performed at a high velocity, causing minimal TUT. Whereas TUT may be one variable to consider for adaptation to resistance exercise, the influence of actual force exerted and barbell displacement also must be considered.

Comparison of the different methods of volume determination between the hypertrophy, strength, and power protocols confirms that different methods of volume determination can yield significantly different results. It is proposed that TW is the most accurate representation of a resistance exercise stimulus because it accounts for actual force produced and barbell displacement. However, it is acknowledged that TW is the most costly and time consuming to calculate in comparison with VL, MDSVL, or TUT. In terms of elucidating the effects of various protocols of resistance exercise, it is recommended that the type of method used for calculation of volume be considered in light of the current evidence.

\section{Practical Applications}

Volume determination is necessary in resistance exercise; however, the methods used to best determine the volume are debatable. The limitations of VL, MDSVL, and TUT as methods for volume determination are evident from the current data. On the basis of the data from the current investigation, TW is the most appropriate method for determining resistance exercise volume. When comparing the effects of different resistance exercise protocols on measures of muscular strength, muscular hypertrophy, and athletic performance, researchers should use TW volume determination in scientific studies. This will ensure that the protocol-specific results in such studies are based on the intensity of the stimuli and not a difference in the TW completed. For the strength and conditioning coach, access to equipment such as force plates and bar-tracking devices may be limited. Thus, if VL, MDSVL, or TUT are used, they should be used with caution when comparing the effectiveness of resistance exercise protocols of different intensities.

\section{REFERENCES}

1. Benson, C, Docherty, D, and Brandenburg, J. Acute neuromuscular responses to resistance training performed at different loads. $J$ Sci Med Sport 9: 135-142, 2006.

2. Campos, GE, Luecke, TJ, Wendeln, HK, Toma, K, Hagerman, FC, Murray, TF, Ragg, KE, Ratamess, NA, Kraemer, WJ, and Staron, RS. Muscular adaptations in response to three different resistancetraining regimens: specificity of repetition maximum training zones. Eur J Appl Physiol 88: 50-60, 2002. 
3. Cormie, P, McBride, JM, and McCaulley, GO. Validation of power measurement techniques in dynamic lower body resistance exercises. J Appl Biomech 23: 103-118, 2007.

4. Cormie, P, McCaulley, GO, and McBride, JM. Power versus strength-power training jump squat training: influence on the loadpower relationship. Med Sci Sports Exerc 39: 996-1003, 2007.

5. Cormie, P, McCaulley, GO, Triplett, NT, and McBride, JM. Optimal loading for maximal power output during lower-body resistance exercises. Med Sci Sports Exerc 39: 340-349, 2007.

6. Crewther, B, Cronin, J, and Keogh, J. Possible stimuli for strength and power adaptation: acute metabolic responses. Sports Med 36: 65-78, 2006.

7. Drinkwater, EJ, Lawton, TW, Lindsell, RP, Pyne, DB, Hunt, PH, and McKenna, MJ. Training leading to repetition failure enhances bench press strength gains in elite junior athletes. J Strength Cond Res 19: 382-388, 2005.

8. Gentil, P, Oliveira, E, and Bottaro, M. Time under tension and blood lactate response during four different resistance training methods. J Physiol Anthropol 25: 339-344, 2006.

9. Gonzalez-Badillo, JJ, Gorostiaga, EM, Arellano, R, and Izquierdo, M. Moderate resistance training volume produces more favorable strength gains than high or low volumes during short-term training cycle. J Strength Cond Res 19: 689-697, 2005.
10. Hunter, GR, Seelhorst, D, and Snyder, S. Comparison of metabolic and heart rate responses to super slow vs. traditional resistance training. J Strength Cond Res 17: 76-81, 2003.

11. Kraemer, JB, Stone, MH, O'Bryant, HS, Conley, MS, Johnson, RL, Nieman, DC, Honeycutt, DR, and Hoke, TP. Effects of single vs. multiple sets of weight training: impact of volume, intensity, and variation. J Strength Cond Res 11: 143-147, 1997.

12. McBride, JM, Blaak, JB, and Triplett-McBride, T. Effect of resistance exercise volume and complexity on EMG, strength, and regional body composition. Eur J Appl Physiol 90: 626-632, 2003.

13. McBride, JM, Triplett-McBride, T, Davie, A, and Newton, RU. The effect of heavy vs. light-load jump squats on the development of strength, power, and speed. J Strength Cond Res 16: 75-82, 2002.

14. McCaulley, GO, Cormie, P, Cavill, MJ, Nuzzo, JL, Urbiztondo, ZG, and McBride, JM. Mechanical efficiency during repetitive vertical jumping. Eur J Appl Physiol 101: 115-123, 2007.

15. Peterson, MD, Rhea, MR, and Alvar, BA. Maximizing strength development in athletes: a meta-analysis to determine the doseresponse relationship. J Strength Cond Res 18: 377-382, 2004.

16. Tran, QT, Docherty, D, and Behm, D. The effects of varying time under tension and volume load on acute neuromuscular responses. Eur J Appl Physiol 98: 402-410, 2006. 